\title{
Las prácticas docentes de los profesores de probabilidad y estadística en las Facultades de Ingeniería
}

Teaching practices of teachers of probability and statistics in the Faculty of Engineering

Pedro Rocha Salamanca y Adriana Patricia Gallego Torres

Universidad Distrital Francisco José de Caldas- Bogota- Colombia. pgrocha@udistrital.edu.co;adpgallegot@udistrital.edu.co

\section{Resumen}

Alrededor de los años setenta se puede observar la inserción de forma explicita y definitiva de los métodos estadísticos en casi todos los programas de ingeniería en el país, esta indusión concuerda con el desarrollo histórico de las ciencias, particularmente la estadística, ya que ésta incipiente disciplina había o estaba sentando las bases teóricas para seleccionar muestras aleatorias, diseñar experimentos, comprobar hipótesis a partir de información observada, analizar datos de tipo cualitativo y cuantitativo y tal vez el mas importante de todos los procesos, permitir la validación de teorías. Esta comunicación se deriva del informe parcial de la tesis doctoral, realizada en el Doctorado en Educación de la Universidad Distrital en el Énfasis de Ciencias. En ella se indaga entre otros: ¿Qué modelo didáctico puede ser utilizado en los espacios de formación, de tal forma que permita la innovación e investigación de las prácticas docentes de los profesores de probabilidad y estadística en las Facultades de Ingeniería para el contexto socio-cultural Bogotano?

\section{Palabras claves}

Prácticas docentes, didáctica de la probabilidad y la estadística

\section{Introduccion}

La confluencia de diferentes acontecimientos de tipo histórico, teórico, y social fortaleció el estudio de la probabilidad, reconociéndose como una altemativa de estudio para muchos fenómenos, ya que esta teoría permitía explicar el comportamiento de una gran cantidad de variables y modelar muchas situaciones. Esta nueva concepción epistemológica permitió la emergencia de nuevos tipos de razonamientos donde la incertidumbre, el azar y la aleatoriedad se encuentran presentes, confrontado la idea de pensamiento determinístico que en muchos espacios académicos la ciencia defendía.

Uno de los resultados de esta inserción en el campo de la educación, se refleja en el hecho que actualmente los diferentes currículos para ingenieros induyen de forma generalizada la enseñanza de los objetos de estudio estocásticos (que están conformados por los objetos estadísticos y probabilísticos), bien como elemento que permite el análisis de datos, la estimación de parámetros, el pronostico de variables, etc. o como soporte conceptual de otras asignaturas o espacios de formación.

Para los profesores igualmente se origino un nuevo problema ya que era necesario enseñar unos nuevos objetos de estudio donde aparece el azar, la incertidumbre, los errores, la simulación, etc. elementos conceptuales de naturaleza muy diferentes a los matemáticos que se enseñaban en las facultades de ingeniería en Colombia. Es muy posible entonces que por su cercanía con las 
matemáticas los docentes adaptaran sus métodos de enseñanza en el desarrollo de sus clases de probabilidad y estadística.

En la misma perspectiva, para los educadores estocásticos, se constituyó entonces un nuevo objeto de estudio de tipo didáctico, que tiene como principal finalidad el mejoramiento del proceso de enseñanza aprendizaje de los conceptos probabilísticos y estadísticos orientados a la ingenieria. Actualmente, son escasas aun las investigaciones sobre cómo es la enseñanza de tales conceptos en el medio Colombiano y sí verdaderamente tales conceptos son utilizados por los ingenieros auando desarrollan su actividad como profesionales o auando toman decisiones utilizando información estadística como ciudadanos, igualmente, no se tiene información de quienes son los responsables de la instrucción estadística en las universidades, de las formas de enseñanza, de las maneras de evaluación, en qué medida las propuestas de enseñanza se han desarrollado, relatando cómo se estructuraron en los diseños curriculares los espacios de formación en estocástica y cómo se han venido transformando en el tiempo, así como las razones de tales evoluciones.

Hoy en día la enseñanza de la estadística, aun no es considera una actividad teóricamente fundamentada, por tanto desconoce los desarrollos históricos producidos y los resultados de la investigación en didáctica de la estocástica, por tanto creemos que la gran mayoría de los profesores enseñan estadística de forma axiomática, de manera tradicional (que se puede entender como una etapa incipiente de resolución de problemas) utilizando metodologías adaptadas de la educación matemática.

Durante los últimos años se han venido desarrollando investigaciones, la mayoría de ellas se encuentran ubicadas en la educación básica y media, pero actualmente, encontramos una inmensa preocupación por parte de los profesores en la educación superior por investigar problemas relacionados con sus practicas docentes en el campo de la estocástica. Pero no solamente en estos espacios los profesores interactúan con el conocimiento estadístico y probabilístico, también es necesario que los profesores cuando realizan investigación en su campo de acción o en educación recurran para analizar, inferir y validar sus resultados a los métodos y teoría estadística, 0 simplemente cuando desean como ciudadanos tomar decisiones en situaciones de incertidumbre objetivando el mundo.

Con los anteriores antecedentes es muy difícil implementar acciones de tipo didáctico que permitan el mejoramiento de los procesos de enseñanza aprendizaje de los objetos de estudio estocásticos. Desde la perspectiva de la acción docente, ya que no es posible en la actualidad para los investigadores en educación estocástica presentar de forma parcial y mucho menos organizada, auáles fueron las principales razones para induir el estudio de los objetos estocásticos, cómo ha sido su desarrollo, qué cambios en las prácticas docentes se han realizado en las fases de diseño, gestión y evaluación en las facultades de ingeniería. Es necesario igualmente estudiar, dasificar, categorizar los métodos que permiten convertir la probabilidad y estadística en una ciencia educacional, estableciendo cuales son sus componentes didácticos espećficos, es decir, los principios teóricos, metodológicos y axiológicos que caracterizan a un docente de estocástica en ingeniería, de tal manera que los profesores puedan mejorar la planeación, gestión y evaluación del proceso de enseñanza aprendizaje de la estocástica.

\section{Preguntas de investigación}

¿Qué modelo didáctico puede ser utilizado en los espacios de formación, de tal forma que permita la innovación e investigación de las prácticas docentes de los profesores de probabilidad y estadística en las Facultades de Ingenieńa para el contexto socio-cultural Bogotano?

Preguntas derivadas 
¿Cuáles fueron las prácticas docentes predominantes en las Facultades de Ingeniería por parte de los profesores, cuando se enfrentaron al problema de enseñar objetos de estudio estocásticos y cuándo aparece la estocástica como una disciplina autónoma en las facultades de ingeniería?

¿Cuáles han sido los principales objetos de estudio estocásticos desplegados en el proceso de enseñanza aprendizaje?

¿Cómo son actualmente las practicas docentes de los profesores de estocástica en las Facultades de Ingeniería?

¿Qué enfoques didácticos caracterizan las prácticas de los profesores?

\section{Axioma I}

Los profesores de estadística en las facultades de ingeniería pueden mejorar sus practicas docentes si;

$>$ Conocen la historia de los objetos de estudio estocásticos y la utilizan en el desarrollo de resolución de problemas en el aula.

$>$ Conocen como han evolucionado las diferentes funciones que realizan los profesores en relación al diseño, ejecución y evaluación del proceso de enseñanza aprendizaje.

$>$ Comprenden, cuestionan y transforman las prácticas docentes y de actuación predominantes en la enseñanza de la probabilidad y estadística actualmente.

> Existe un modelo didáctico que utilice los resultados de las investigaciones en educación estocástica para la enseñanza de la probabilidad y estadística.

\section{Algunos referentes teoricos}

El presente trabajo se enmarca dentro de un campo espećfico, que se denominara Didáctica de la Estocástica la cual inicialmente pienso que se compone de dos grandes campos de enseñanza el primero relaciona todos los elementos conceptuales de la teoría de la probabilidad y el segundo corresponde a los diferentes métodos estadísticos.

Según Rocha (2006) uno de los principales objetivos de la didáctica de la estocástica cuando se entiende como campo de investigación es el estudio de las interrelaciones entre los componentes que inciden en los procesos de enseñanza aprendizaje de los objetos de estudio estocásticos. Este tipo de

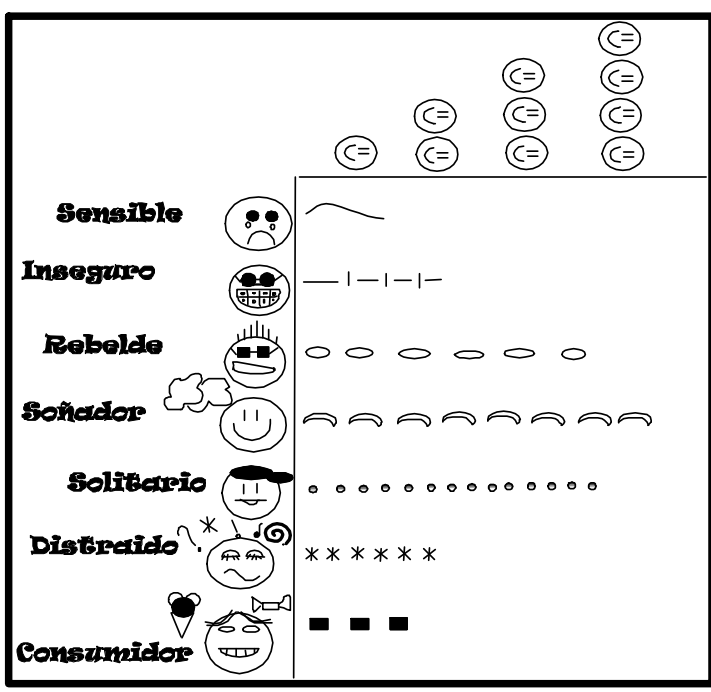
interrelaciones son de naturaleza difusa ya que se encuentran afectadas por variados factores (sociales, culturales, cognitivo, etc.) la filosofía del conocimiento científico permite determinar aules son estas interrelaciones sus características y ontología.

Algunos de los objetos de estudio por tanto de la didáctica de la estocástica son:

1. El origen o historia sobre como se han desplegado las diferentes teorías que existen en didáctica de la estocástica.

2. La génesis de los objetos de estudio estocásticos.

3. El estudio del denominado Sistema didáctico, que relaciona el conocimiento del profesor, del estudiante y el epistemológico, en un entomo espećfico.

4. La teoría y la investigación en la enseñanza de la probabilidad y estadística.

5. Los modelos que permiten el diseño de unidades didácticas en probabilidad y estadística que puedan ser desarrolladas en el aula, lo que Chevallard denomino Ingeniería didáctica.

6. Las prácticas de los profesores de estadística en el aula.

7. Las interacciones en los procesos de enseñanza aprendizaje. 
8. Las rutas de estudio y aprendizaje de los estudiantes.

9. Las dificultades en la enseñanza y aprendizaje de los objetos de estudio estocástico.

10. El diseño curricular en probabilidad y estadística.

11. La formación de profesores de estocástica.

12. La evaluación de los procesos.

Un profesor se enfrenta con varios problemas cuando desea enseñar algún objeto de estudio que se relaciona con el denominado conocimiento prácticol, el primero tiene que ver con la relación que crean entre el conocimiento estocástico a enseñar (polo epistemológico), el conocimiento estocástico que aprendieron en el desanollo como profesores (o profesionales) y el estado del conocimiento estocástico de sus estudiantes (polo cognitivo). En segundo lugar la importancia y utilidad que le atribuyen al denominado conocimiento práctico del profesor $y$ por último lo que saben en relación a los procesos a desamollar en sus estudiantes.

Al abordar este campo de estudio nos enfrentamos con un conjunto de interrogantes de mucha importancia como por ejemplo:

$>$ ¿Cómo perciben los estudiantes el mundo de la incertidumbre?

$>$ ¿Qué concepciones tienen los estudiantes sobre las nociones básicas del conocimiento probabilística?

Cómo interpretan y utilizan los estudiantes la información estadística?

En este trabajo se desarrolla solamente el denominado polo didáctico o del profesor.

Al referirse a la práctica docente del profesor se está expresando el conjunto de estrategias didácticas y mediaciones instrumentales que el profesor pone en juego en el aula para construir significados sobre los objetos matemáticos a partir de la interacción entre él y sus estudiantes 0 entre éstos.

\section{La noción de historia de la educación estocástica.}

Después de los años setenta los investigadores en Educación en Colombia en concordancia con los eventos ocurridos a nivel mundial han venido cuestionando, evaluando y revisando, los supuestos teóricos que sustentan su epistemología, metodología, ideología etc. y que subyacen dentro de las diferentes teorías pedagógicas desarrolladas. Este movimiento no ha sido exclusivo de las ciencias de la educación, como afirma Cabrera (2005) existe "un creciente cuestionamiento crítico, durante los últimos años a algunos de los más arraigados presupuestos teóricos, epistemológicos y filosóficos en que se habían asentado largamente, con anterioridad en la investigación histórica.[...] La consecuencia de este avestionamiento critico ha sido la emergencia de una nueva perspectiva historiográfica que, aunque aún presenta contornos algo borrosos, es ya daramente visible en la obra un grupo significativo de historiadores y de teóricos de la historia". (Pág.22).

De igual manera existen de forma general diferentes objetivos de realización en el estudio de la historia de cualquier objeto de estudio, en tomo a esta idea Viñado (2005) propone los siguientes:

> "Descubrir y mostrar la presencia del pasado en los debates, auestiones y problemas educativos de su tiempo, es decir, no sólo hacer la genealogía del presente, sino también desvelar los usos incorrectos del pasado, $y$ sobre todo las apropiaciones, mitificaciones y manipulaciones del mismo

\footnotetext{
${ }^{1}$ Entendemos como Guerrero y Sánchez por conocimiento práctico del profesor aquel conocimiento que pone en juego con respecto a lo que él sabe sobre las matemáticas escolares, del como la aprendieron y acerca del como se enseña.
} 
> Afirmar y mostrar su papel potencial, desde una perspectiva genealógica y critica, en el estudio $y$ comprensión de la educación $y$ de los problemas $y$ cuestiones contemporáneas, [...]. La historia de la educación debe, en este sentido, arrojar luz sobre el presente, ayudar a entenderlo y a situarse en el mismo.

> Hacer público el pasado de modos diferentes, en función de las audiencias, haciendo pública el mismo tiempo su actividad y su existencia a fin de ser testigos de su tiempo, dar testimonio del mismo e ímaginar el futuro ayudando a construirlo.

$>$ Reafimar y reforzar, para todo ello, la naturaleza profesional, sus instrumentos de análisis y estudio." Pág. 153

Este apartado presenta sin la pretensión de ser exhaustivos algunos elementos que enmarcan la postura epistemológica del trabajo enmarcado dentro de la Educación Estocástica, delimitado como investigación histónica del desarrollo objetos de estudio estocásticos en las facultades de ingenieńa.

En la delimitación del objeto de estudio se utilizara como referente teórico el propuesto por Laspalas (2002) auando afirma "si aplicamos la distinción dásica entre objeto 'material' y objeto 'formal' de una ciencia a la historia de la educación, tenemos que el objeto 'material' de ésta aquello que intenta conocer- es la 'educación', mientras que su objeto'formal' - la perspectiva dedes la cual lo observa- es la 'histórica'. Así el objetivo de nuestra disciplina es analizar la educación desde su dimensión histórica". (Pág.86). Transfiriendo la anterior definición se podría suponer que puede existir entonces una disaiplina denominada historia de la Educación Estocástica en las facultades de ingeniería que tendría entre otros los siguientes objetos de estudio:

$>$ El desarrollo histórico del pensamiento estocástico pedagógico, que puede ser entendido como el estudio de los componentes que permiten la construcción en los individuos de razonamiento de tipo probabilístico y estadístico.

$>$ Los cambios en el tiempo ocurridos en la actividad docente cuando los profesores enseñan estadística y probabilidad.

> Las políticas educativas que han incidido dentro de las reformas de tipo curricular.

$>$ La génesis o historia del surgimiento de los objetos de estudio estocásticos.

$>$ Las diferentes instituciones educativas es decir, el entomo bajo el cual tiene lugar el proceso de enseñanza aprendizaje.

$>$ Las diferentes propuestas didácticas que pueden ser utilizadas en la enseñanza de la probabilidad y estadística.

En la educación superior existe la tendencia en las facultades de distribuir el estudio de los objetos estadísticos casi siempre en dos cursos después de haberse realizado la instrucción en matemáticas y durante los primeros semestres, en el primero se desarrolla en la mayoría de los casos las temáticas relacionadas con la estadística descriptiva (distribuciones de frecuencia, medidas de tendencia central y dispersión, medidas de localización; percentiles cuartiles, medidas de forma; simetría, curtosis) y la teoría de la probabilidad (enfoques de probabilidad frecuencial, dásica o Laplaciana, axiomas y teoremas de probabilidad, independencia de eventos, probabilidad condicional, conjunta y marginal, variables aleatorias y distribuciones de probabilidad, esperanza matemática, distribuciones de probabilidad discreta y continua), en el segundo se desarrolla la estadística inferencial, pero tratando de realizar el trabajo en el aula con niveles de complejidad superiores. Así mismo, es reaurrente la inclusión dentro del segundo curso de algunos métodos estadísticos que permiten estimar parámetros y realizar pronósticos (distribuciones muestrales, comprobación de hipótesis, intervalos de confianza, análisis de varianza, modelos lineales, etc) que son requeridos para el desarrollo curricular en semestres posteriores. Por último, en algunos planes de estudio, dependiendo de su naturaleza se encuentran algunas asignaturas que son necesarias para el desarrollo conceptual de los estudiantes, y que están directamente relacionadas con el razonamiento estadístico (muestreo, psicometría, econometría, didáctica de la estadística, series de tiempo teoría bayesiana, métodos no parametricos, control de calidad, etc.) 
El anterior panorama es solo parcial, ya que presenta únicamente la perspectiva de los contenidos, sin embargo es necesario considerar algunos problemas estructurales que no permiten actualmente ver de forma compleja el estado del conocimiento de los estudiantes y la problemática de la enseñanza de la probabilidad y estadística.

\section{Metodología}

Se puede considerar que el enfoque metodológico de la investigación es de tipo; teórico holístico, descriptivo y explicativo, por tanto los métodos etnográficos pueden ser de esencial utilización.

Dentro del enfoque etnográfico se consideran los datos como una información potencialmente verificable extraída del entomo. Teniendo en cuenta lo anterior, las categorías de estrategias de recogida de datos más empleadas en etnografía son la observación, las entrevistas, los instrumentos diseñados por el investigador, señalan Goetz \& Lecompte (1988). Para dar respuesta a las preguntas de investigación utilizaremos también una metodología de corte cualitativo. Este enfoque permitirá hacer descripciones sobre la educación. El diseño metodológico contempla diversas fases; 1. Construcción teórico metodológica, 2. Diseño de instrumentos de indagación, 3. Recolección de información, 4. Estudio estadístico para los instrumentos de indagación, 5. Triangulación de los díferentes instrumentos de indagación. 6. Análisis y verificación de los resultados obtenidos. 7. Socialización de los resultados, 8. Publicación. Consideramos que algunos de los instrumentos serán; las observaciones de las dases de los profesores de estadística en el salón de dase que serán video grabadas; entrevistas a los profesores participantes las cuales serán grabadas en audio y transcritas para facilitar el análisis; y recolección de algunos instrumentos que los profesores usan en sus dases

\section{Resultados Esperados}

Se espera poder dar respuesta a las preguntas de investigación planteadas: comparar lo que es y lo que debe ser la educación estocástica en facultades de ingeniería del país, describir el conocimiento pedagógico del profesor de estocástica, describir la historiografía de los objetos estocásticos, los recursos de apoyo que el profesor de usa en su dase y sus concepciones. Esperamos contribuir al campo de la educación estocástica a través de la realización y publicación de la tesis doctoral y de su presentación en congresos nacionales e internacionales.

\section{Bibliogragóa}

Cabrera, M. (2005) La crisis de la modemidad y la renovación de los estudios históricos en Repensar la historia de la Educación Pág. 22 - 52 Editorial Biblioteca Nueva Madrid España.

Goetz, J. y LeCompte, M. (1988). Etnografía y diseño aualitativo en investigación educativa. Ed. Morata Madrid

Laspalas, J. (2002) Introducción a la historiografía de la educación Ediciones Universidad de Navarra España.

Rocha P., (2006). Cuademos de investigación. No. 10. Educación Estocástica.: Universidad Distrital "Francisco José de Caldas". Bogotá, Colombia

Viñao A. (2005) La historia de la Educación ante el siglo XXI: tensiones, reos y audiencias. Compilador Ferraz . L M. en Repensar la Historia de la Educación. (2005) Editorial Biblioteca Nueva Madrid España 